\title{
@creative
}

Also available at http://amc-journal.eu

ISSN 1855-3966 (printed edn.), ISSN 1855-3974 (electronic edn.)

ARS MATHEMATICA CONTEMPORANEA 9 (2015) 77-91

\section{Minimal covers of equivelar toroidal maps*}

\author{
Kostiantyn Drach ${ }^{\dagger}$ \\ V.N. Karazin Kharkiv National University, School of Mathematics and Mechanics, \\ Geometry Department, Svobody Sq. 4, 61022, Kharkiv, Ukraine \\ Mark Mixer \\ Wentworth Institute of Technology, Department of Applied Mathematics \\ 550 Huntington Ave., Boston, MA 02115
}

Received 8 November 2012, accepted 29 April 2014, published online 11 July 2014

\begin{abstract}
Given any equivelar map on the torus, it is natural to consider its covering maps. The most basic of these coverings are finite toroidal maps or infinite tessellations of the Euclidean plane. In this paper, we prove that each equivelar map on the torus has a unique minimal toroidal rotary cover and also a unique minimal toroidal regular cover. That is to say, of all the toroidal rotary (or regular) maps covering a given map, there is a unique smallest. Furthermore, using the Gaussian and Eisenstein integers, we construct these covers explicitly.
\end{abstract}

Keywords: Minimal covers, Regular and rotary maps, Gaussian and Eisenstein integers.

Math. Subj. Class.: 52B15, 51M20, 52C22

\section{Introduction}

The classification of regular and chiral maps on the torus was first published in 1948 by Coxeter [3]. Recently, other classifications of toroidal maps have been presented, e.g. [1], [6], [11]. It is known that every equivelar toroidal map has a universal covering, in that it can be covered by a regular tessellation of the Euclidean plane. However, equivelar toroidal maps can be covered by smaller maps that also have high degrees of symmetry. Notably, each equivelar toroidal map has a unique finite minimal regular cover (see [7], [14]).

Recently, there has been much interest in finding such minimal regular covers for different families of maps and abstract polytopes (see for example [8, 9, 17]). Our paper is

\footnotetext{
${ }^{\dagger}$ Partially supported by the Akhiezer Foundation and I. E. Tarapov Scholarship.

E-mail addresses: drach@karazin.ua (Kostiantyn Drach), mixerm@wit.edu (Mark Mixer)
} 
part of this broader project of understanding minimal covers. However, we focus not only on regular covers, where much is known, but also on rotary covers (those that are either regular or chiral).

In this paper we give a proof that any equivelar toroidal map has a unique minimal regular cover on the torus. While this result follows from the previously mentioned work on, our techniques allow for the explicit construction of these covers using the Gaussian and Eisenstein integers. Furthermore, we prove a similar result, that each such map is uniquely covered by a finite minimal rotary toroidal map, and again using number theoretic results, we can construct these covers.

Our main results are the summarized by the following theorem.

Theorem 1.1. Each equivelar map on the torus has a unique minimal rotary cover on the torus, and a unique minimal regular cover on the torus. Each can be constructed explicitly.

An unpublished construction of these minimal regular covers was also made by Maksym Skoryk using an application of the theory of linear Diophantine equations. However, in this paper, we use a different approach, which yields the construction of minimal regular covers. Additionally, our construction provides the more general result concerning minimal rotary covers.

The paper is organized as follows. In section 2 we give necessary definitions and state results about maps and covers. Section 3 consists mainly of relevant number theoretic results regarding the Gaussian and the Eisenstein integers. This section concludes with the technical statement of two theorems, which together prove Theorem 1.1. The following two sections provide the proof of these two theorems, where Section 4 deals with minimal rotary covers and Section 5 considers regular covers. Finally, in order to illustrate our results, we construct such minimal covers for some families of equivelar toroidal maps in Section 6.

\section{Maps and Covers}

In this section we provide some definitions and results that will be of use in the future; most of these ideas, as well as further details, can be found in $[2,13,18]$.

A finite graph $X$ embedded on a compact 2-dimensional manifold $S$ such that every connected component of $S \backslash X$ (which is called a face) is homeomorphic to an open disc is called a map $\mathcal{M}$ (on the surface $S$ ). An automorphism of $\mathcal{M}$ is an automorphism of the underlying graph $X$ that can be extended to a homeomorphism of the surface $S$. The automorphisms of a map $\mathcal{M}$ form a group, which is called the automorphism group, denoted $\operatorname{Aut}(M)$. Note that although any map is symmetric under uncountably many homeomorphisms for the underlying surface $S$, we adopt a purely combinatorial (hence finite) point of view. A map $\mathcal{M}$ is equivelar of (Schläfli) type $\{p, q\}$ if all its vertices are $q$-valent and all its faces are topological $p$-gons.

Definition 2.1. Let $\mathcal{M}$ and $\mathcal{N}$ be maps on the surfaces $S$ and $S^{\prime}$ respectively. A surjective function $\eta: S^{\prime} \rightarrow S$ that preserves adjacency and sends vertices to vertices, edges to edges, and faces to faces of the maps $\mathcal{M}$ and $\mathcal{N}$ is called a covering of the map $\mathcal{M}$ by the map $\mathcal{N}$. This is denoted by $\mathcal{N} \searrow \mathcal{M}$, and we say that $\mathcal{N}$ is a cover of $\mathcal{M}$.

It follows from Theorem 4.14 of [15], that any equivelar map $\mathcal{M}$ is covered by the universal map $\mathcal{U}$ of the same type $\{p, q\}$, and that $\mathcal{M}$ is a quotient of $\mathcal{U}$ by some subgroup of the automorphism group of $\mathcal{U}$. 
If $\mathcal{M}$ and $\mathcal{N}$ are both of type $\{p, q\}$ then, it can be easily shown from the above definition that there is a constant number $K$ (possibly infinite) of faces of $\mathcal{N}$ that are sent to any face of $\mathcal{M}$. This follows directly from the connectedness of the maps and the adjacency preserving property of a covering. Furthermore, this number $K$ is also the number of edges or vertices of $\mathcal{N}$ that are sent to an edge or vertex of $\mathcal{M}$. In this case, we say that $\mathcal{N} \searrow \mathcal{M}$ is a $K$-sheeted covering.

Hereafter, we consider equivelar maps of type $\{p, q\}$ on a 2-dimensional torus $\mathbb{T}^{2}$, which are simply called equivelar toroidal maps. On the torus the only possible types to consider are $\{4,4\},\{3,6\}$, and $\{6,3\}$. However, since any map of type $\{6,3\}$ is dual to one of type $\{3,6\}$, and a cover of the dual is the dual of a cover, we will only consider maps of type $\{4,4\}$, and $\{3,6\}$ in this paper.

Equivelar toroidal maps can be seen as quotients of regular tessellations of the Euclidean plane. Given a regular tessellation $\tau$ of the plane, we denote the group of translations that preserve the tessellation as $T_{\tau}$. For each group $T_{\tau}$, we can pick two shortest linearly independent translations $\mathbf{e}_{\mathbf{1}}, \mathbf{e}_{\mathbf{2}}$ which generate $T_{\tau}$. Furthermore, in this paper $\mathbf{e}_{1}, \mathbf{e}_{2}$ are chosen with equal length and with the angle between them equal to $\frac{\pi}{2}$ for $\{4,4\}$, or $\frac{\pi}{3}$ for $\{3,6\}$. We call $\left(\mathbf{e}_{\mathbf{1}}, \mathbf{e}_{\mathbf{2}}\right)$ the basis connected with the tessellation $\tau$. Hereafter, for each tessellation we fix the basis connected with it and will consider coordinates of all other vectors in the plane with respect to this basis.

The next theorem gives an essential characterization of all equivelar toroidal maps.

Theorem 2.2. [16] Let $\mathcal{M}$ be an equivelar map on the torus. Then $\mathcal{M}$ can be obtained as a quotient of a regular tessellation $\tau$ of the Euclidean plane by some translation subgroup $G<T_{\tau}$ generated by two linearly independent vectors. That is to say, $\mathcal{M}=\tau / G$.

This theorem shows that there is a one-to-one correspondence between equivelar toroidal maps of type $\{p, q\}$ and translation subgroups of $T_{\tau}$ generated by two non-collinear vectors, where $\tau$ is the regular tessellation of the plane of type $\{p, q\}$. We also point out that the converse of this theorem is obvious; any map on the torus that is obtained as a quotient of a regular tessellation by a translation subgroup is an equivelar map. We use as a standard notation $\tau_{\mathbf{a}, \mathbf{b}}:=\tau /\langle\mathbf{a}, \mathbf{b}\rangle$ to indicate a map obtained as a quotient of a plane regular tessellation $\tau$ by a subgroup $\langle\mathbf{a}, \mathbf{b}\rangle\left\langle T_{\tau}\right.$.

A flag of a planar tessellation $\tau$ is a triple of an incident vertex, edge, and face of the tessellation. We then define a flag of a map $\tau_{\mathbf{a}, \mathbf{b}}$ as the orbit of a flag under the group $\langle\mathbf{a}, \mathbf{b}\rangle$. When the map is combinatorially equivalent to an abstract polytope (see [13]), this is equivalent to a flag equalling a triple of an incident vertex, edge, and face of the map itself. Two flags of a map on the torus are said to be adjacent if they lift to flags in the plane that differ in exactly one element.

Definition 2.3. A map $\mathcal{M}$ is called regular if and only if $\operatorname{Aut}(M)$ acts transitively on the set of flags. A map $\mathcal{M}$ is called chiral if $\operatorname{Aut}(M)$ has two orbits on flags with adjacent flags lying in different orbits.

Definition 2.4. A map $\mathcal{M}$ is called rotary if it is either regular or chiral.

We note here that in the classical work of Coxeter (see [4], originally in [3]) rotary maps are called regular, whereas regular and chiral in our terminology were reflexible and irreflexible respectively following Coxeter's terminology. All regular and rotary toroidal maps with the type $\{p, q\}$ can be easily described in terms of their corresponding translation subgroups. 
Theorem 2.5. [3, 4] A toroidal map $\mathcal{M}$ of type $\{4,4\}$ is rotary if and only if $\mathcal{M}=$ $\{4,4\} /\langle(s, t),(-t, s)\rangle$ for some $s, t \in \mathbb{Z}, s^{2}+t^{2} \neq 0$. A toroidal map of type $\{3,6\}$ is rotary if and only if $\mathcal{M}=\{3,6\} /\langle(s, t),(-t, s+t)\rangle$ for some $s, t \in \mathbb{Z}, s^{2}+t^{2} \neq 0$. Additionally, such maps are regular if and only if $\operatorname{st}(s-t)=0$.

It follows from Theorem 2.5 that in order to obtain a rotary toroidal map of type $\{p, q\}$ we must take a quotient of a regular tessellation of the same type by a subgroup generated by vectors $\mathbf{a}$ and $\mathbf{b}$ of equal length, and with the angle between $\mathbf{a}$ and $\mathbf{b}$ equal to $\pi / 2$ for type $\{4,4\}$ or equal to $\pi / 3$ for type $\{3,6\}$.

If we let $\mathcal{M}, \mathcal{N}$ be equivelar toroidal maps such that $\mathcal{M}$ is a quotient of a plane regular tessellation $\tau$ by its translation subgroup $G<T_{\tau}$, it follows from this definition that $\mathcal{N} \searrow$ $\mathcal{M}$ if and only if there is a subgroup $H<G$ such that $\mathcal{N}=\tau / H$. Additionally, if $H$ is a subgroup of $G=\langle\mathbf{a}, \mathbf{b}\rangle$, then $H=\langle\mathbf{u}, \mathbf{v}\rangle$, where

$$
\begin{aligned}
& \mathbf{u}=n_{1} \mathbf{a}+m_{1} \mathbf{b}, \\
& \mathbf{v}=n_{2} \mathbf{a}+m_{2} \mathbf{b},
\end{aligned}
$$

for some integers (not all zero) $n_{1}, n_{2}, m_{1}, m_{2}$, with $n_{1} m_{2} \neq n_{2} m_{1}$. For more details on this, see Sections 3 and 4 of [15]. Thus, for equivelar toroidal maps $\mathcal{N}=\tau / H, \mathcal{M}=\tau / G$, $H<G$, it follows that if $\mathcal{N} \searrow \mathcal{M}$ is a $K$-sheeted covering, then the number $K$ of sheets can be easily found from the representation in (2.1) as follows:

$$
K=\left|n_{1} m_{2}-n_{2} m_{1}\right| \text {. }
$$

The expression in (2.2) is simply obtained by comparing the area of the parallelogram spanned by $\mathbf{u}, \mathbf{v}$ to that spanned by $\mathbf{a}, \mathbf{b}$.

Let us also consider the covering from another perspective. For a map $\mathcal{M}=\tau / G$, where $G=\langle\mathbf{a}, \mathbf{b}\rangle$, we call the parallelogram spanned by the vectors $\mathbf{a}, \mathbf{b}$ a fundamental region of $\mathcal{M}$. Then, a fundamental region for the covering map $\mathcal{N}=\tau / H$ can be viewed as $K$ fundamental regions of $\mathcal{M}$ glued together (see figure 1 ). It is easy to show that the number $K$ of sheets is equal to the index $[G: H]$ of the groups generating the cover $\tau / H \searrow \tau / G$.

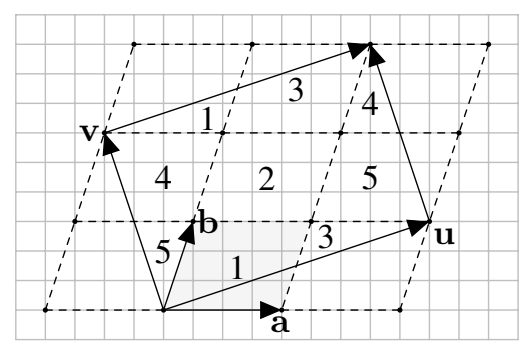

Figure 1: $\{4,4\}_{\mathbf{u}, \mathbf{v}} \searrow\{4,4\}_{\mathbf{a}, \mathbf{b}}$ is a 5 -sheeted covering, and the covering map $\{4,4\}_{\mathbf{u}, \mathbf{v}}$ is obtained by gluing together 5 fundamental regions of $\{4,4\}_{\mathbf{a}, \mathbf{b}}$

Definition 2.6. [9], [13] An equivelar toroidal map $\mathcal{N}$ is a minimal rotary (regular) toroidal cover of an equivelar toroidal map $\mathcal{M}$ if $\mathcal{N}$ is a rotary (regular) map, $\mathcal{N} \searrow \mathcal{M}$, and for any other rotary (regular) equivelar toroid $\mathcal{L}$ such that $\mathcal{N} \searrow \mathcal{L} \searrow \mathcal{M}$ it follows that $\mathcal{L}=\mathcal{N}$ or $\mathcal{L}=\mathcal{M}$. 


\section{The Gaussian and Eisenstein integers}

The Gaussian and Eisenstein integers provide an essential tool for constructing minimal covers for equivelar toroidal maps. Plotting these domains in the complex plane produces precisely the vertex set of a regular tessellation: $\tau=\{4,4\}$ for the Gaussian integers and $\tau=\{3,6\}$ for the Eisenstein integers. Since we use the Gaussian and Eisenstein integers to construct minimal covering maps, we must recall some of their properties in this section. We will follow [12].

The Gaussian integers $\mathbb{Z}[i]$ are defined as $\{a+b i \mid a, b \in \mathbb{Z}\}$, where $i=\sqrt{-1}$. Similarly, the Eisenstein integers $\mathbb{Z}[\omega]$ are defined as $\{a+b \omega \mid a, b \in \mathbb{Z}\}$, where $\omega=$ $(1+i \sqrt{3}) / 2$. Hereafter, we write $\mathbb{Z}[\sigma], \sigma=i, \omega$, to denote either of these two sets.

In this paper we use the following notation: given $\alpha=a+b \sigma \in \mathbb{Z}[\sigma]$, we call its conjugate the number $\bar{\alpha}:=a+b \bar{\sigma}$, where $\bar{\sigma}$ is the conjugate complex number to $\sigma \in \mathbb{C}$. Also we call $\operatorname{Re} \alpha:=a$ and $\operatorname{Im} \alpha:=b$ the real and imaginary parts respectively. Note here that if $\sigma=i$, then this is the traditional notion of 'real part', and 'imaginary part' of a complex number. However, if $\sigma=\omega$, then the 'traditional' real and imaginary parts of $a+b \omega$ are $a+b / 2$ and $b \sqrt{3} / 2$, respectively.

It is straightforward to prove the following technical lemma, which we will need later.

Lemma 3.1. For every $\alpha, \beta \in \mathbb{Z}[\sigma]$ the following holds:

(1) $\operatorname{Im}(\bar{\alpha} \beta)=\operatorname{Re} \alpha \operatorname{Im} \beta-\operatorname{Im} \alpha \operatorname{Re} \beta$;

(2) $\operatorname{Im}(\bar{\alpha} \beta)=-\operatorname{Im}(\alpha \bar{\beta})$.

For every $\alpha=a+b \sigma \in \mathbb{Z}[\sigma]$, we assign the norm $N(\alpha):=\alpha \bar{\alpha}$. We recall that this is a multiplicative function; namely, for $\alpha, \beta \in \mathbb{Z}[\sigma], N(\alpha \beta)=N(\alpha) N(\beta)$. A number $\alpha \in \mathbb{Z}[\sigma] \backslash\{0\}$ divides $\beta \in \mathbb{Z}[\sigma]$ if and only if there is $\gamma \in \mathbb{Z}[\sigma]$ such that $\beta=\alpha \gamma$. In this case we will use the notation $\alpha \mid \beta$. Recall that, in the ring of Gaussian integers, the units are only $\pm 1, \pm i$, while in the ring of Eisenstein integers the units are only $\pm 1, \pm \omega, \pm \bar{\omega}$.

Two numbers $\alpha, \beta \in \mathbb{Z}[\sigma]$ are associated $(\alpha \simeq \beta$ ) iff $\alpha=\beta \varepsilon$ for some unit $\varepsilon$. It is easy to show that $\simeq$ is an equivalence relation. A number $\pi \in \mathbb{Z}[\sigma]$ is a prime in the ring $\mathbb{Z}[\sigma]$ if and only if it is not a unit and $\alpha \mid \pi$ implies that $\alpha$ is either a unit or associated with $\pi$. Equivalently, $\pi \in \mathbb{Z}[\sigma]$ is a prime if and only if it is not a unit and from $\pi \mid(\alpha \beta)$ it follows that $\pi \mid \alpha$ or $\pi \mid \beta$.

The Gaussian and the Eisenstein integers are unique factorization domains and thus there is a theorem analogous to the fundamental theorem of arithmetic for these rings.

Theorem 3.2 (Unique Factorization Theorem, [12]). Let $\mathbb{Z}[\sigma]$ be the ring of Gaussian or Eisenstein integers and let $\mathbb{P}_{\sigma} \subset \mathbb{Z}[\sigma]$ be a set of primes in $\mathbb{Z}[\sigma]$ such that every prime number $\pi \in \mathbb{Z}[\sigma]$ is associated with a unique prime $\pi^{\prime} \in \mathbb{P}_{\sigma}$, so that none of the numbers in $\mathbb{P}_{\sigma}$ is associated with any other. Then for every $\alpha \in \mathbb{Z}[\sigma], \alpha \neq 0$ there exists a unique representation:

$$
\alpha=\varepsilon \pi_{1}^{e_{1}} \ldots \pi_{n}^{e_{n}}
$$

where $\varepsilon$ is a unit, $\pi_{1}, \ldots, \pi_{n} \in \mathbb{P}_{\sigma}$ are pairwise distinct prime numbers, and $e_{1}, \ldots, e_{n} \in$ $\mathbb{N}$ are natural numbers.

Additionally, it is possible to describe all the primes in $\mathbb{Z}[\sigma]$ in terms of their norms. For the Gaussian integers $\mathbb{Z}[i]$ there are the following possible types of primes $\pi$ :

(1) $\pi \simeq 1+i, N(\pi)=2$. Since $\overline{1+i}=\bar{\imath}(1+i), \bar{\pi}$ is associated with $\pi$; 
(2) rational prime numbers $q \equiv 3(\bmod 4)$. As $q \in \mathbb{Z}$ its conjugate $\bar{q}=q, \bar{q}$ is associated with $q$;

(3) $\pi \in \mathbb{Z}[i]$ such that $N(\pi)=p$ is an rational prime $p \equiv 1(\bmod 4)$. Note that in this case $\bar{\pi}$ is a prime which is not associated with $\pi$.

For the Eisenstein ring $\mathbb{Z}[\omega]$ there are similar types of primes:

(1) $\pi \simeq 1+\omega ; N(\pi)=3, \bar{\pi} \simeq \pi$;

(2) rational prime numbers $q \equiv 2(\bmod 3), \bar{q}=q$;

(3) $\pi \in \mathbb{Z}[\omega]$ such that $N(\pi)=p$ is an rational prime $p \equiv 1(\bmod 3)$. $\bar{\pi}$ is a prime not associated with $\pi$.

The following two lemmas follow from the Unique Factorization Theorem and the classification of the primes. The proofs for the both of this lemmas are straightforward and, therefore, omitted.

Lemma 3.3. Let $\alpha \in \mathbb{Z}[\sigma] \backslash\{0\}$ and $\alpha=\pi_{0}^{a_{0}} q_{1}^{a_{1}} \ldots q_{s}^{a_{s}} \pi_{1}^{b_{1}} \ldots \pi_{t}^{b_{t}}$ be its prime decomposition, where $\pi_{0} \simeq 1+\sigma$, for $j \in\{1, \ldots s\}$ the numbers $q_{j}$ are rational primes of type (2), for $k \in\{1, \ldots t\}$ the numbers $\pi_{k}$ are primes of type (3), and all exponents are nonnegative integers. Then the following are equivalent:

(1) $\operatorname{GCD}(\operatorname{Re} \alpha, \operatorname{Im} \alpha)=1$;

(2) $a_{0} \in\{0,1\}, a_{1}=\ldots=a_{s}=0$ and $\pi_{j} \not 4 \bar{\pi}_{k}$ for all $k, j \in\{1, \ldots, t\}$.

Lemma 3.4. Let $\alpha \in \mathbb{Z}[\sigma] \backslash\{0\}$. Then $(1+\sigma) \mid \alpha$ if and only if $\operatorname{Re} \alpha \equiv \operatorname{Im} \alpha(\bmod N(1+$ $\sigma))$.

A complex number $\gamma \in \mathbb{Z}[\sigma]$ is a greatest common divisor (GCD) of $\alpha, \beta \in \mathbb{Z}[\sigma]$, $N(\alpha)+N(\beta) \neq 0$, if $\gamma \mid \alpha$ and $\gamma \mid \beta$ and for every $\gamma^{\prime} \in \mathbb{Z}[\sigma]$ such that $\gamma^{\prime} \mid \alpha$ and $\gamma^{\prime} \mid \beta$ it follows $\gamma^{\prime} \mid \gamma$. From the Unique Factorization Theorem it follows that a greatest common divisor is well defined up to associates. Thus, we can consider the GCD equivalence class $[\operatorname{GCD}(\alpha, \beta)]$.

Further, we use the common notation $\gamma=\operatorname{GCD}(\alpha, \beta)$ implying that $\gamma \in[\operatorname{GCD}(\alpha, \beta)]$. If there is a rational integer $n \in[\operatorname{GCD}(\alpha, \beta)]$, then we specifically take $\operatorname{GCD}(\alpha, \beta):=|n|$. For example, $[\operatorname{GCD}(3,6 i)]=\{3,-3,3 i,-3 i\}$, and thus $\operatorname{GCD}(3,6 i)=3$.

As we have seen, either the Gaussian or the Eisenstein integers can be linked with a regular tessellation $\tau$. Then the numbers 1 and $\sigma$ from $\mathbb{Z}[\sigma]$ correspond to the basis $\left(\mathbf{e}_{1}, \mathbf{e}_{2}\right)$ of translations connected with $\tau$, and the operations of addition and multiplication by an integer in $\mathbb{Z}[\sigma]$ can be seen as the same operations in $T_{\tau}$ treated as a vector space over $\mathbb{Z}$ with the basis $\left(\mathbf{e}_{\mathbf{1}}, \mathbf{e}_{\mathbf{2}}\right)$. From this point of view, we will identify every two vectors $\mathbf{a}=\left(a_{1}, a_{2}\right)$, $\mathbf{b}=\left(b_{1}, b_{2}\right)$ with the complex numbers $\alpha:=a_{1}+a_{2} \sigma \in \mathbb{Z}[\sigma]$ and $\beta:=b_{1}+b_{2} \sigma \in \mathbb{Z}[\sigma]$. Finally, using this idea, we will denote $\tau_{\alpha, \beta}:=\tau_{\mathbf{a}, \mathbf{b}}$. Furthermore we let $\tau_{\eta}:=\tau_{\eta, \sigma \eta}$.

It follows from Theorem 2.5 that, if $\eta \in \mathbb{Z}[\sigma] \backslash\{0\}$, then $\tau_{\eta}$ is a rotary toroidal map, and all rotary maps can be obtained in this way. Note that if $\eta \simeq \eta^{\prime}$, then $\tau_{\eta}=\tau_{\eta^{\prime}}$. This implies that we need only look for a generator $\eta$ of a rotary map $\tau_{\eta}$ up to associates. Additionally, a rotary map $\tau_{\eta}$ is regular iff $\eta \simeq n$ or $\eta \simeq n(1+\sigma)$, where $n \in \mathbb{Z} \backslash\{0\}$.

Using the notation developed in this section, we are now ready to state the formal versions of the two theorems which together yield the main theorem of this paper. The proofs of these two theorems are the content of the next two sections. We remind the reader of our convenient but non-standard definition for $\operatorname{Im} \gamma$ when $\gamma \in \mathbb{Z}[\omega]$. 
Theorem 3.5. Let $\tau_{\alpha, \beta}$ be an equivelar toroidal map represented as a quotient of a regular planar tessellation $\tau$ by a translation subgroup $\langle\alpha, \beta\rangle<T_{\tau}$ generated by two non-collinear vectors, corresponding to the complex numbers $\alpha, \beta \in \mathbb{Z}[\sigma]$. Let $\gamma=\operatorname{GCD}(\alpha, \beta)$. There exists a unique minimal rotary covering map equal to $\tau_{\eta_{\text {min }}}$ with

$$
\eta_{\text {min }}=\frac{\operatorname{Im}(\bar{\alpha} \beta)}{N(\gamma)} \gamma .
$$

Moreover, the number $K_{\text {min }}$ of fundamental regions of $\tau_{\alpha, \beta}$ glued together in order to obtain $\tau_{\eta_{\text {min }}}$ is equal to

$$
K_{\text {min }}=\frac{|\operatorname{Im}(\bar{\alpha} \beta)|}{N(\gamma)} .
$$

Theorem 3.6. Let $\tau_{\alpha, \beta}$ be an equivelar toroidal map represented as a quotient of a regular planar tessellation $\tau$ by a translation subgroup $\langle\alpha, \beta\rangle<T_{\tau}$ generated by two non-collinear vectors, corresponding to the complex numbers $\alpha, \beta \in \mathbb{Z}[\sigma]$. Let $c=\operatorname{GCD}(\operatorname{Re} \alpha, \operatorname{Im} \alpha$, $\operatorname{Re} \beta, \operatorname{Im} \beta)$. Then for $\tau_{\alpha, \beta}$ there exists a unique minimal regular covering map $\tau_{\eta_{\text {min }}}$ with

$$
\eta_{\text {min }}= \begin{cases}\frac{\operatorname{Im}(\bar{\alpha} \beta)}{N(1+\sigma) c}(1+\sigma), & \text { if } \frac{\operatorname{Re} \alpha}{c} \equiv \frac{\operatorname{Im} \alpha}{c} \text { and } \frac{\operatorname{Re} \beta}{c} \equiv \frac{\operatorname{Im} \beta}{c} \bmod N(1+\sigma) ; \\ \frac{\operatorname{Im}(\bar{\alpha} \beta)}{c}, & \text { otherwise. }\end{cases}
$$

Moreover, the number $K_{\text {min }}$ of fundamental regions of $\tau_{\alpha, \beta}$ glued together in order to obtain $\tau_{\eta_{\text {min }}}$ is equal to

$$
K_{\text {min }}= \begin{cases}\frac{|\operatorname{Im}(\bar{\alpha} \beta)|}{N(1+\sigma) c^{2}}, & \text { if } \frac{\operatorname{Re} \alpha}{c} \equiv \frac{\operatorname{Im} \alpha}{c} \text { and } \frac{\operatorname{Re} \beta}{c} \equiv \frac{\operatorname{Im} \beta}{c} \bmod N(1+\sigma) ; \\ \frac{|\operatorname{Im}(\bar{\alpha} \beta)|}{c^{2}}, & \text { otherwise. }\end{cases}
$$

\section{Proof of Theorem 3.5}

In this section we give the proof of our first main theorem, which states that every equivelar map on the torus has a unique minimal rotary cover on the torus. To accomplish this proof, we translate the concepts of covers from Section 2 into the terminology developed in Section 3, and construct all possible toroidal rotary covers for a given map. By doing so, we can explicitly find a cover that is minimal.

Let $\tau_{\alpha, \beta}$ be an equivelar toroidal map represented as a quotient of a regular planar tessellation $\tau$ by a translation subgroup $\langle\alpha, \beta\rangle<T_{\tau}$ generated by two non-collinear vectors, corresponding to complex numbers $\alpha, \beta \in \mathbb{Z}[\sigma]$.

We have seen that each rotary map can be described as $\tau_{\eta}$ for some $\eta \in \mathbb{Z}[\sigma]$. Additionally, we have seen that covering maps satisfy the conditions of (2.1). Thus, in order to obtain a rotary map $\tau_{\eta}$ which covers $\tau_{\alpha, \beta}$, we need to find a number $\eta \in \mathbb{Z}[\sigma] \backslash\{0\}$ such that:

$$
\left\{\begin{array}{l}
n_{1} \alpha+m_{1} \beta=\sigma \eta, \\
n_{2} \alpha+m_{2} \beta=\eta,
\end{array}\right.
$$

for some integers $n_{1}, m_{1}, n_{2}, m_{2}$, with $n_{1} m_{2} \neq n_{2} m_{1}$. Let $\nu=-n_{1}+n_{2} \sigma$ and $\mu=$ $m_{1}-m_{2} \sigma$. we must find $\nu, \mu \in \mathbb{Z}[\sigma], N(\nu)+N(\mu) \neq 0$, where (using the notation of Section 3): 


$$
\left\{\begin{aligned}
-\alpha \operatorname{Re} \nu+\beta \operatorname{Re} \mu & =\sigma \eta \\
\alpha \operatorname{Im} \nu-\beta \operatorname{Im} \mu & =\eta .
\end{aligned}\right.
$$

We proceed by solving system (4.2) considering $\eta$ as a parameter. From the possible solutions to this system, we can determine the exact value of $\eta$ which generates the minimal cover $\tau_{\eta_{\text {min }}} \searrow \tau_{\alpha, \beta}$.

Multiplying the second equation by $\sigma$, and subtracting the first from it; we get the following equation

$$
\alpha \nu-\beta \mu=0 \text {. }
$$

Let $\gamma:=\operatorname{GCD}(\alpha, \beta)$. Then $\alpha / \gamma, \beta / \gamma \in \mathbb{Z}[\sigma]$, and $\operatorname{GCD}(\alpha / \gamma, \beta / \gamma)=1$. From this and (4.3) it follows that

$$
\nu=\frac{\beta}{\gamma} \delta, \mu=\frac{\alpha}{\gamma} \delta,
$$

for some $\delta \in \mathbb{Z}[\sigma] \backslash\{0\}$. In order to also be a solution to system (4.2) it is sufficient that $\gamma \mid \eta$, and thus $\eta / \gamma \in \mathbb{Z}[\sigma]$. To solve system (4.2) we simply substitute (4.4) into the second equation from (4.2). This yields:

$$
\frac{\alpha}{\gamma} \operatorname{Im}\left(\frac{\beta}{\gamma} \delta\right)-\frac{\beta}{\gamma} \operatorname{Im}\left(\frac{\alpha}{\gamma} \delta\right)=\frac{\eta}{\gamma} .
$$

After multiplying by $\delta$ and taking the imaginary and real parts of both sides, we can use both parts of Lemma 3.1 to get that:

$$
\begin{aligned}
& \operatorname{Im}\left(\frac{\eta}{\gamma} \delta\right)=0 \\
& \operatorname{Re}\left(\frac{\eta}{\gamma} \delta\right)=N(\delta)\left(\frac{\operatorname{Im}(\bar{\alpha} \beta)}{N(\gamma)}\right) .
\end{aligned}
$$

Therefore,

$$
\eta=\left(\frac{\operatorname{Im}(\bar{\alpha} \beta)}{N(\gamma)}\right) \bar{\delta} \gamma
$$

Thus, $\nu=\beta / \gamma \cdot \delta$ and $\mu=\alpha / \gamma \cdot \delta$ is a solution to system (4.2), and a rotary map $\tau_{\eta}$ covers $\tau_{\alpha, \beta}$ if and only if the parameter $\eta$ satisfies (4.5). Notice here that $\frac{\operatorname{Im}(\bar{\alpha} \beta)}{N(\gamma)} \in \mathbb{Z}$.

Let us show that the unique minimal rotary cover is generated by the complex number (up to associates)

$$
\eta_{\text {min }}=\frac{\operatorname{Im}(\bar{\alpha} \beta)}{N(\gamma)} \gamma .
$$

Indeed, observe that for any $\delta_{1}, \delta_{2} \in \mathbb{Z}[\sigma] \backslash\{0\}$ we have $\tau_{\delta_{1} \delta_{2}} \searrow \tau_{\delta_{1}}$. Therefore, for any $\delta \in \mathbb{Z}[\sigma] \backslash\{0\}$ and the corresponding complex number $\eta$ satisfying (4.5), the map $\tau_{\eta}$ covers $\tau_{\eta_{\min }}$. Then from definition (2.6) it follows that $\tau_{\eta_{\min }}$ is a minimal cover of $\tau_{\alpha, \beta}$. Even more, if $\delta$ is not a unit, then the corresponding rotary map $\tau_{\eta} \neq \tau_{\eta_{\text {min }}}$. Thus, $\tau_{\eta_{\text {min }}}$ is a unique minimal cover. The first part of Theorem 3.5 is proved.

Let us find the number $K_{\min }$ of fundamental regions of $\tau_{\alpha, \beta}$ that we should glue together in order to obtain $\tau_{\eta_{\text {min }}}$. From (2.2), for an arbitrary cover $\tau_{\eta}$ substituting our 
solutions for $\mu$ and $\nu$, this number is equal to

$$
\begin{aligned}
K & =\left|m_{1} n_{2}-m_{2} n_{1}\right|=|\operatorname{Re} \mu \operatorname{Im} \nu-\operatorname{Re} \nu \operatorname{Im} \mu|=|\operatorname{Im}(\bar{\mu} \nu)| \\
& =\left|\operatorname{Im}\left(\frac{\bar{\alpha}}{\bar{\gamma}} \bar{\delta} \frac{\beta}{\gamma} \delta\right)\right|=\frac{|\operatorname{Im}(\bar{\alpha} \beta)|}{N(\gamma)} N(\delta) .
\end{aligned}
$$

Finally, since for the minimal cover $\delta \simeq 1$, we obtain

$$
K_{\text {min }}=\frac{|\operatorname{Im}(\bar{\alpha} \beta)|}{N(\gamma)} .
$$

This completes the proof of Theorem 3.5.

\section{Proof of Theorem 3.6}

In this section we give the proof of our second main theorem, which describes how every equivelar map on the torus has a unique minimal regular cover on the torus. Let $\tau_{\alpha, \beta}$ be an equivelar toroidal map represented as a quotient of a regular planar tessellation $\tau$ by a translation subgroup $\langle\alpha, \beta\rangle<T_{\tau}$ generated by two non-collinear vectors, corresponding to complex numbers $\alpha, \beta \in \mathbb{Z}[\sigma]$.

It follows from Theorem 2.5 that in order to produce any regular cover on the torus $\tau_{\eta} \searrow \tau_{\alpha, \beta}$ we need to take a quotient of a regular tessellation $\tau$ by a translation subgroup $\langle\eta, \sigma \eta\rangle<T_{\sigma}$ with $\eta \simeq n$ or $\eta \simeq n(1+\sigma), n \in \mathbb{Z} \backslash\{0\}$.

To proceed, we separately construct the families of regular covers of each type (depending on $\eta$ ), and determine which type produces the minimal cover.

I. Let $\eta \simeq n, n \in \mathbb{Z} \backslash\{0\}$. Since we are looking for a value of $\eta$ up to associates, we may assume $\eta \in \mathbb{Z}$. Then as $\frac{\operatorname{Im}(\bar{\alpha} \beta)}{N(\gamma)} \in \mathbb{Z}$, from (4.5) it follows that $\bar{\delta} \gamma \in \mathbb{Z}$. Writing $\gamma=c \gamma_{1}$, where $c=\operatorname{GCD}(\operatorname{Re} \gamma, \operatorname{Im} \gamma)$, we have $\operatorname{GCD}\left(\operatorname{Re} \gamma_{1}, \operatorname{Im} \gamma_{1}\right)=1$, and from Lemma 3.3 it follows that $\delta$ is equal $k \gamma_{1}$ for some $k \in \mathbb{Z} \backslash\{0\}$. Thus, regular covers of this type have the form $\tau_{\eta_{I}}$ for

$$
\eta_{I}=\frac{\operatorname{Im}(\bar{\alpha} \beta)}{c} k,
$$

where $k \in \mathbb{Z} \backslash\{0\}$ is an arbitrary non-zero integer.

II. Let $\eta \simeq n(1+\sigma), n \in \mathbb{Z} \backslash\{0\}$. As in the previous case, (4.5) implies that $\eta \simeq$ $n(1+\sigma)$ if and only if $\bar{\delta} \gamma \simeq n_{1}(1+\sigma)$ for some $n_{1} \in \mathbb{Z} \backslash\{0\}$. Since $\overline{1+\sigma} \simeq 1+\sigma$, we have $(1+\sigma)^{2 k} \simeq(N(1+\sigma))^{k}$.

Thus, we should consider two subcases: $\gamma=(1+\sigma)^{2 k+1} \gamma^{\prime}$ and $\gamma=(1+\sigma)^{2 k} \gamma^{\prime}$, where $k$ is a positive integer and $(1+\sigma) \not \gamma^{\prime}$.

(IIa) Let $\gamma=(1+\sigma)^{2 k+1} \gamma^{\prime}$. Then $\bar{\delta} \gamma \simeq n_{1}(1+\sigma)$ if and only if $\bar{\delta}(1+\sigma)^{2 k} \gamma^{\prime}$ is associated with a non-zero integer. As noted above, $(1+\sigma)^{2 k} \simeq(N(1+\sigma))^{k}$. Additionally, if $\gamma^{\prime}=d \gamma^{\prime \prime}$ and $d=\operatorname{GCD}\left(\operatorname{Re} \gamma^{\prime}, \operatorname{Im} \gamma^{\prime}\right)$, then $\bar{\delta}(1+\sigma)^{2 k} \gamma^{\prime}$ is associated with a non-zero integer if and only if $\delta \simeq \gamma^{\prime \prime} l$ for some $l \in \mathbb{Z} \backslash\{0\}$. Note that if $c=\operatorname{GCD}(\operatorname{Re} \gamma, \operatorname{Im} \gamma)$, then $c=(N(1+\sigma))^{k} d$.

Therefore, regular covers of this subcase have the form $\tau_{\eta_{I I a}}$ for

$$
\eta_{I I a}=\frac{\operatorname{Im}(\bar{\alpha} \beta)}{N(1+\sigma) c}(1+\sigma) l,
$$


where $l \in \mathbb{Z} \backslash\{0\}$ is arbitrary.

(IIb) Let $\gamma=(1+\sigma)^{2 k} \gamma^{\prime}$. Then $\bar{\delta} \gamma \simeq n_{1}(1+\sigma), n_{1} \in \mathbb{Z} \backslash\{0\}$, if and only if $\overline{\left(\frac{\delta}{1+\bar{\sigma}}\right)} \gamma$ is associated with a non-zero integer. As above, if $\gamma=c \gamma_{1}, c=\operatorname{GCD}(\operatorname{Re} \gamma, \operatorname{Im} \gamma)$, then $K$ is minimal if and only if $\frac{\delta}{1+\bar{\sigma}} \simeq \gamma_{1}$. Thus using (4.5), we find that regular covering maps $\tau_{\eta_{I I b}}$ for this subcase are provided by

$$
\eta_{I I b}=\frac{\operatorname{Im}(\bar{\alpha} \beta)}{c}(1+\sigma) l,
$$

for an arbitrary non-zero integer $l$.

Using the observation at the end of Section 4, we now can identify a minimal regular cover in families (5.1) - (5.3). Note that we should consider either the cases I and IIa, or I and IIb.

We claim in subcase IIa that the minimal regular cover $\tau_{\eta_{\text {min }}}$ is generated by

$$
\eta_{\text {min }}=\frac{\operatorname{Im}(\bar{\alpha} \beta)}{N(1+\sigma) c}(1+\sigma) .
$$

Indeed, it is obvious that $\tau_{\eta_{I I a}} \searrow \tau_{\eta_{\text {min }}}$ for any $l$ in $\eta_{I I a}$ and $\tau_{\eta_{I I a}} \neq \tau_{\eta_{\text {min }}}$ unless $l=1$. Furthermore, since $\eta_{I}=\eta_{\min } \cdot(\overline{(1+\sigma)} k)$ for any non-zero integer $k$ corresponding to (5.1), we have $\tau_{\eta_{I}} \searrow \tau_{\eta_{\text {min }}}$. Thus, the claim holds.

Turning to subcase IIb, the unique minimal regular cover cover $\tau_{\eta_{\min }}$ is likewise generated by

$$
\eta_{\min }=\frac{\operatorname{Im}(\bar{\alpha} \beta)}{c} .
$$

The last thing we should notice here is that

$$
c=\operatorname{GCD}(\operatorname{Re} \gamma, \operatorname{Im} \gamma)=\operatorname{GCD}(\operatorname{Re} \alpha, \operatorname{Im} \alpha, \operatorname{Re} \beta, \operatorname{Im} \beta) .
$$

This follows from the observation that if $k \in \mathbb{Z}, k \neq 0$ and $\alpha \in \mathbb{Z}[\sigma] \backslash\{0\}$, then $k \mid \alpha$ if and only if $k \mid \operatorname{Re} \alpha$ and $k \mid \operatorname{Im} \alpha$.

Finally, applying Lemma 3.4 to distinguish between the subcases and computing the corresponding numbers $K_{\min }$ of fundamental regions using (4.6), we finish the proof of Theorem 3.6.

\section{Examples}

To illustrate our theorems, we now build the minimal rotary and minimal regular covering maps for different families of equivelar toroidal maps. Our examples are motivated by the classification of equivelar toroidal maps by symmetry type (found in [11]; a detailed classification of 2- and 4-orbit toroids was first studied in [10]).

\subsection{Maps of type $\{4,4\}$}

It is clear that the minimal rotary and minimal regular covering map of a regular map is just the map itself. For a chiral map, the minimal rotary cover also coincides with the initial map; however the minimal regular cover differs. Furthermore, given a map in any of the other families of 2-orbit toroidal maps, the minimal regular cover is equal to the minimal rotary cover. In particular, for integers $a>b>0$, we have the following four cases. 
Example 6.1. For a map $\{4,4\}_{(a, 0),(0, b)}$, we get $\alpha=a, \beta=b i, \gamma=\operatorname{GCD}(a, b i)=$ $\operatorname{GCD}(a, b)=c,|\operatorname{Im}(\bar{\alpha} \beta)|=a b$. Thus the minimal regular (and rotary) cover is $\{4,4\}_{\frac{a b}{c}}$, which can be obtained by gluing $\frac{a b}{c^{2}}$ fundamental regions of the initial map.

Example 6.2. For a map $\{4,4\}_{(a, b),(a,-b)}$, we get $\alpha=a+b i, \beta=a-b i, c=\operatorname{GCD}(a, b)$, $|\operatorname{Im}(\bar{\alpha} \beta)|=2 a b$. Since $\bar{\alpha}=\beta$, then $\gamma=c$ if $\frac{a}{c} \not \equiv \frac{b}{c}(\bmod 2)$, or $\gamma=c(1+i)$ if $\frac{a}{c} \equiv \frac{b}{c}(\bmod 2)$. In the first case the minimal regular (and rotary) cover is $\{4,4\}_{\frac{2 a b}{c}}$, and the number of fundamental regions is equal to $\frac{2 a b}{c^{2}}$. In the second case the minimal regular (and rotary) cover is $\{4,4\}_{\frac{a b}{c}(1+i)}$, where the number of fundamental regions of the initial toroidal map we glued together in order to obtain this cover is equal to $\frac{a b}{c^{2}}$.

Example 6.3. For a map $\{4,4\}_{(a, b),(b, a)}$ the situation is similar to Example 6.2. We have $\alpha=a+b i, \beta=b+a i, c=\operatorname{GCD}(a, b)$, and $|\operatorname{Im}(\bar{\alpha} \beta)|=a^{2}-b^{2}$. Since $i \bar{\alpha}=\beta$, we get that $\gamma=c$ if $\frac{a}{c} \not \equiv \frac{b}{c}(\bmod 2)$, or $\gamma=c(1+i)$ otherwise. Thus, the minimal regular (and rotary) cover is built in the same manner as in Example 6.2.

Example 6.4. For the final family of 2 -orbit equivelar toroidal maps $\{4,4\}_{(a, a),(b,-b)}$, we get $\alpha=a+a i, \beta=b-b i, c=\operatorname{GCD}(a, b), \gamma=c(1-i)$, and $|\operatorname{Im}(\bar{\alpha} \beta)|=2 a b$. Thus, the minimal regular (and rotary) cover is $\{4,4\}_{\frac{a b}{c}(1+i)}$, which can be obtained by gluing $\frac{a b}{c^{2}}$ fundamental regions of the initial map (see Figure 2 (b)).

Given a 4-orbit toroidal map, the minimal regular cover and the minimal rotary cover may or may not coincide; this depends on $\gamma$. For example, the minimal regular and minimal rotary covers of the map $\{4,4\}_{(2,1),(7,0)}$ coincide. On the other hand, the covers of $\{4,4\}_{(4,3),(5,0)}=\{4,4\}_{(4,3),(-1,3)}$ are distinct (see Figure $2(\mathrm{a})$ ).

\subsection{Maps of type $\{3,6\}$}

The situation for regular and chiral toroidal maps of type $\{3,6\}$ is similar to the $\{4,4\}$ case. Let us instead consider the families of 3 -orbit maps of type $\{3,6\}$. Let $a, b \in \mathbb{N}$.

Example 6.5. For a 3-orbit toroidal map $\{3,6\}_{(a, 0),(-b, 2 b)}$, we have that $\alpha=a, \beta=$ $-b+2 b \omega, c=\operatorname{GCD}(a, b)$, and $|\operatorname{Im}(\bar{\alpha} \beta)|=2 a b$. Since $\beta=b \omega(1+\omega)$, we know that $\gamma=c$ if $\frac{a}{c} \not \equiv 0(\bmod 3)$ and $\gamma=c(1+\omega)$ otherwise. This implies that the minimal regular (and rotary) cover is equal to $\{3,6\}_{\frac{2 a b}{c}}$, where the number of fundamental regions glued to obtain the cover is $\frac{2 a b}{c^{2}}$ in the first case; and $\{3,6\}_{\frac{2 a b}{3 c}(1+\omega)}$, where the number of fundamental regions is equal to $\frac{2 a b}{3 c^{2}}$, in the second case.

Example 6.6. For a 3 -orbit map $\{3,6\}_{(a, b),(a+b,-b)}$, we write that $\alpha=a+b \omega, \beta=$ $a+b-b \omega, c=\operatorname{GCD}(a, b)$, and $|\operatorname{Im}(\bar{\alpha} \beta)|=2 a b+b^{2}$. Since $\bar{\alpha}=\beta$, we get that $\gamma=c$ if $\frac{a}{c} \not \equiv \frac{b}{c}(\bmod 3)$ and $\gamma=c(1+\omega)$ otherwise. Thus, the minimal regular (and rotary) cover is $\{3,6\}_{\frac{2 a b+b^{2}}{c}}$, where the number of fundamental regions is equal to $\frac{2 a b+b^{2}}{c^{2}}$ in the first case; and $\{3,6\}_{\frac{2 a b+b^{2}}{3 c}(1+\omega)}$, where the number of fundamental regions is $\frac{2 a b+b^{2}}{3 c^{2}}$ in the second case (see Figure 3(b)).

As in the last subsection, the 6-orbit toroidal maps of type $\{3,6\}$ have minimal regular and minimal rotary covers which may or may not coincide. Again this depends on $\gamma$ (see Figure 3). 


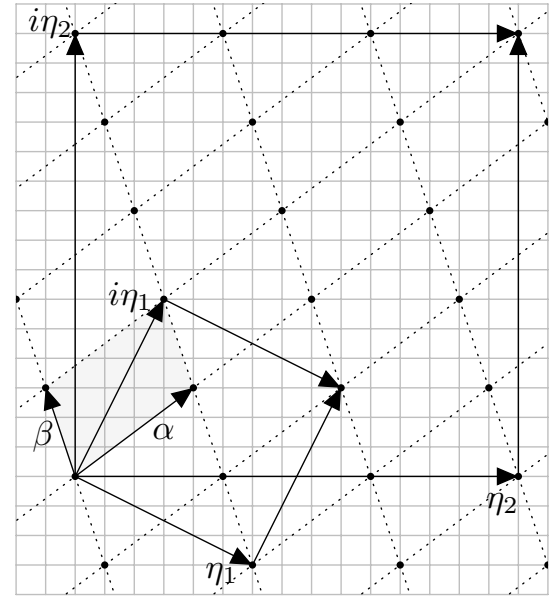

(a)

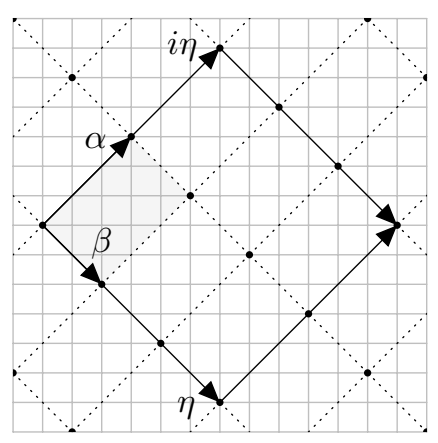

(b)

Figure 2: (a) For the 4-orbit toroidal map $\mathcal{M}=\{4,4\}_{\alpha, \beta}, \alpha=4+3 i, \beta=-1+3 i$ the complex number $\eta_{1}=6-3 i$ generates the unique minimal rotary covering toroidal map $\{4,4\}_{6-3 i}$, which can be obtained by gluing 3 fundamental regions of the initial map. Meanwhile, the unique minimal regular covering toroidal map for $\mathcal{M}$ is $\{4,4\}_{15}$, generated by $\eta_{2}=15$, can be obtained by gluing 15 fundamental regions. (b) For the 2-orbit toroidal map $\{4,4\}_{\alpha, \beta}, \alpha=3+3 i, \beta=2-2 i$ the minimal rotary and regular covering toroidal maps coincide with the regular toroidal map $\{4,4\}_{\eta}, \eta=6-6 i$, which can be obtained by gluing together 6 fundamental regions of the initial map. 


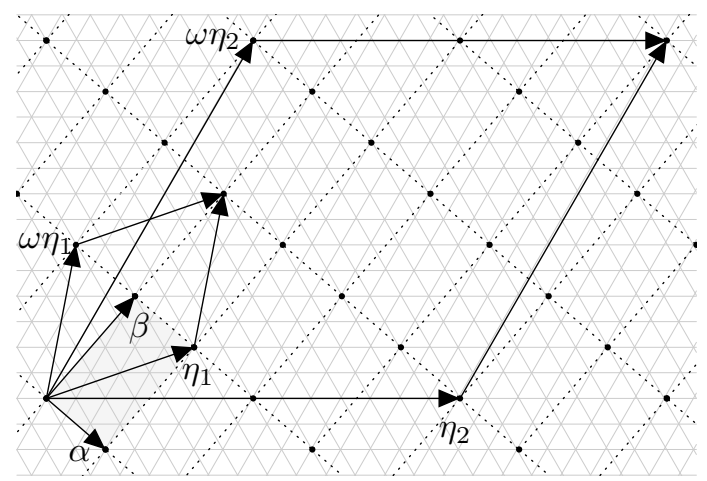

(a)

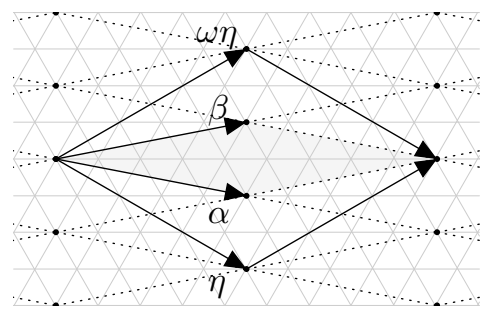

(b)

Figure 3: (a) For the 6-orbit toroidal map $\mathcal{M}=\{3,6\}_{\alpha, \beta}, \alpha=3-2 \omega, \beta=1+4 \omega$ the unique minimal rotary covering toroidal map is $\{3,6\}_{\eta_{1}}$ with $\eta_{1}=4+2 \omega$, which can be obtained by gluing 2 fundamental regions of the initial map. Meanwhile, the unique minimal regular covering map for $\mathcal{M}$ is $\{3,6\}_{\eta_{2}}$ with $\eta_{2}=14$, obtained by gluing 14 fundamental regions. (b) For the 3-orbit toroidal map $\{3,6\}_{\alpha, \beta}, \alpha=5-\omega, \beta=4+\omega$ the minimal rotary and regular covers coincide and are equal to $\{3,6\}_{\eta}, \eta=6-3 \omega$, which can be obtained by gluing 3 fundamental regions. 


\section{Concluding remarks}

As noted in Section 1, this paper is part of a larger project which seeks to understand the structure of highly symmetric covers of various maps and polytopes. While many of the results in this area have concerned minimal regular covers, there are other natural questions to consider.

For example, if instead we are given a map on the torus which is not equivelar, the minimal regular and rotary covers will not be maps on the torus. Instead, it is natural to try and construct toroidal covers of these maps which have the greatest possible symmetry. Such a question is being considered in a natural successor of this paper (see [5]).

\section{Acknowledgments}

Much of this work was done while the authors were involved in the Fields-MITACS 2011 Summer Undergraduate Research Program. We would like to express our deep gratitude to the Fields Institute for the hospitality and the creative atmosphere. We also would like to thank Daniel Pellicer, Isabel Hubard, and Asia Weiss for their constant support, and for providing the question which has been solved in this paper. The first named author also would like to thank Maksym Skoryk and Alexander Borisenko for fruitful discussions. Both authors thank the anonymous referees for many valuable remarks and improvements.

\section{References}

[1] U. Brehm and W. Kühnel, Equivelar maps on the torus, European J. Combin. 29 (2008), 18431861.

[2] U. Brehm and E. Schulte, Polyhedral maps, in: J. Goodman, J. O'Rourke (eds.), Handbook of discrete and computational geometry, CRC Press Ser. Discrete Math. Appl., Boca Raton, 1997, 345-358.

[3] H. S. M. Coxeter, Configurations and maps, Rep. Math. Colloq. (2) 8 (1948), 18 - 38.

[4] H. S. M. Coxeter and W. O. J. Moser, Generators and Relations for Discrete Groups, 3rd ed., Springer-Verlag, Berlin, 1972.

[5] K. Drach and M. Skoryk, Archimedean toroids and their minimal almost regular covers (to appear).

[6] R. Duarte, 2-restrictedly-regular hypermaps of small genus, PhD Thesis, University of Aveiro, 2007.

[7] M. I. Hartley, All polytopes are quotients, and isomorphic polytopes are quotients by conjugate subgroups, Discrete Comput. Geom. 21 (1999), 289-298.

[8] M. I. Hartley and G. I. Williams, Representing the sporadic Archimedean polyhedra as abstract polytopes, Discrete Math. 31012 (2010), 1835-1844.

[9] M. I. Hartley, D. Pellicer and G. Williams, Minimal covers of the prisms and antiprisms, Discrete Math. 31220 (2012), 3046-3058.

[10] I. Hubard, Two-orbit polyhedra from groups, J. Combin. 31 (2010), 943-960.

[11] I. Hubard, A. Orbanić, D. Pellicer and A. I. Weiss, Symmetries of equivelar 4-toroids, Discrete Comput. Geom. 48 (2012), 1110-1136.

[12] K. Ireland and M. Rosen, A classical introduction to modern number theory, 2nd ed., SpringerVerlag, New-York, 1990. 
[13] P. McMullen and E. Schulte, Abstract Regular Polytopes, Encyclopedia of Math. Appl., vol. 92, Cambridge University Press, Cambridge, 2002.

[14] B. Monson, D. Pellicer and G. Williams, The Tomotope Ars Math. Contemp. 5 (2012), 355 370.

[15] B. Monson, D. Pellicer and G. Williams, Mixing and Monodromy of Abstract Polytopes. Trans. Amer. Math. Soc. 366 (2014), 2651-2681.

[16] S. Negami, Uniqueness and faithfullness of embedding of toroidal graphs, Discrete Math. 44 (1983), 161-180.

[17] D. Pellicer and G. Williams, Minimal covers of the Archimedean tilings, Part I, Electron. J. Comb. 19 (2012).

[18] J. Širáň, Regular maps on a given surface: a survey, in: M. Klazar, J. Kratochvil (eds.), Topics in Discrete Mathematics. Algorithms and Combinatorics, vol. 26, Springer, Berlin, 2006, 591609.

[19] J. Tits, A local approach to buildings, in: C. Davis, B. Grünbaum (eds.), The Geometric Vein: the Coxeter Festschrift, Springer-Verlag, NewYork - Heidelberg - Berlin, 1981, 519-547. 